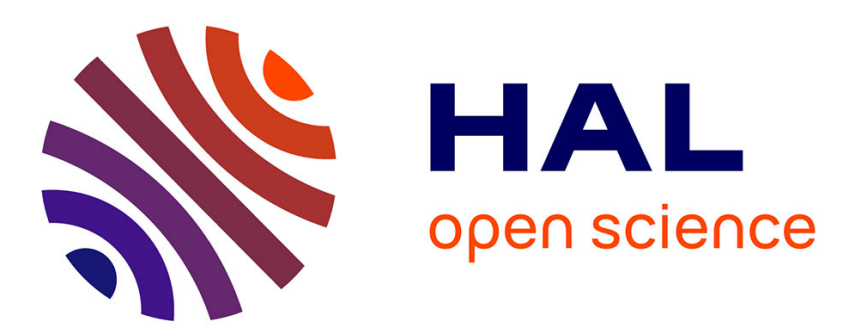

\title{
Continuous-time model identification from noisy input/output measurements using fourth-order cumulants
}

Stéphane Thil, Hugues Garnier, Marion Gilson, Kaushik Mahata

\section{- To cite this version:}

Stéphane Thil, Hugues Garnier, Marion Gilson, Kaushik Mahata. Continuous-time model identification from noisy input/output measurements using fourth-order cumulants. 46th IEEE Conference on Decision and Control, CDC'07, Dec 2007, New Orleans, United States. pp.CDROM. hal-00167446

\author{
HAL Id: hal-00167446 \\ https://hal.science/hal-00167446
}

Submitted on 20 Aug 2007

HAL is a multi-disciplinary open access archive for the deposit and dissemination of scientific research documents, whether they are published or not. The documents may come from teaching and research institutions in France or abroad, or from public or private research centers.
L'archive ouverte pluridisciplinaire HAL, est destinée au dépôt et à la diffusion de documents scientifiques de niveau recherche, publiés ou non, émanant des établissements d'enseignement et de recherche français ou étrangers, des laboratoires publics ou privés. 


\title{
Continuous-time model identification from noisy input/output measurements using fourth-order cumulants
}

\author{
Stéphane Thil*, Hugues Garnier*, Marion Gilson*, Kaushik Mahata**
}

\begin{abstract}
In this paper, the problem of identifying stochastic linear continuous-time systems from noisy input/output data is addressed. The input of the system is assumed to be non-Gaussian, whereas the noises contaminating the data are assumed to be Gaussian. The fourth-order cumulants of the input/output data are then (asymptotically) insensitive to the noises, that can be colored and/or mutually correlated. Two estimators based on this noise-cancellation property are proposed. The performance of the proposed algorithms are assessed through a numerical simulation.
\end{abstract}

\section{INTRODUCTION}

Errors-in-variables (EIV) system identification - where the input and the output of the system are contamined by noises - has received considerable interest in the last few years. Many methods have been proposed to solve the EIV problem for discrete-time models, see [19], [17] for an overview. Nonetheless, in many areas of science and engineering, the identified dynamic models should be physically meaningful. As a result, there is a need for modeling approaches that are able to yield directly from the sampled data parsimonious continuous-time (CT) models that have clear physical interpretations. Although the last few years have witnessed considerable development in CT approaches to system identification from sampled data (see e.g. [9], [11], [16], [8]), CT model identification in an EIV framework is a relatively unexplored area. The first attempts have been very recently proposed [13], [18], assuming the noises contaminating the data to be white. This assumption allows not only to simplify the algorithms, but to rule out some identifiability problems as well. Indeed, without any further assumptions on the signal and noise models, it is wellknown that the general EIV model is not uniquely identifiable from second order statistics [2], [1]. EIV systems suffer from this lack of identifiability, and it is thus of interest to study alternative approaches based on higher-order statistics. Several identification methods using higher-order statistics have been proposed for discrete-time EIV models (see e.g. [7], [24], [6], [25]).

A CT EIV system identification method using third-order cumulants has been recently proposed [23]. The use of third-order cumulants, which are insensitive to symmetrically distributed noises, allows to achieve improved performance in low signal-to-noise conditions. Furthermore, the method

\footnotetext{
* Centre de Recherche en Automatique de Nancy, Nancy-Université, CNRS, Faculté des Sciences et Techniques, BP 239, 54506 Vandœuvre-lèsNancy Cedex, France. Email: firstname.lastname@cran.uhp-nancy.fr

** Centre for Complex Dynamic Systems and Control, School of Electrical Engineering and Computer Science, University of Newcastle, Callaghan, NWS 2308, Australia. Email: kaushik.mahata@newcastle.edu.au
}

can be applied as long as the noises are symmetrically distributed: the noises on both input and output signals can thus be colored, and even mutually correlated. However, a restriction is that the noise-free input signal must have a skewed probability density function. To circumvent this restriction, fourth-order cumulants can be used.

This paper presents a fourth-order cumulant-based method for CT system identification in an EIV framework. The paper is organized in the following way. The identification problem is formulated in Section II. The main definitions and properties of higher-order statistics used in the proposed approach are then recalled in Section III. The fourth-order statistics-based algorithms for continuous-time EIV models are presented in Section IV. A numerical study is presented in Section V before concluding in Section VI.

\section{Problem Statement}

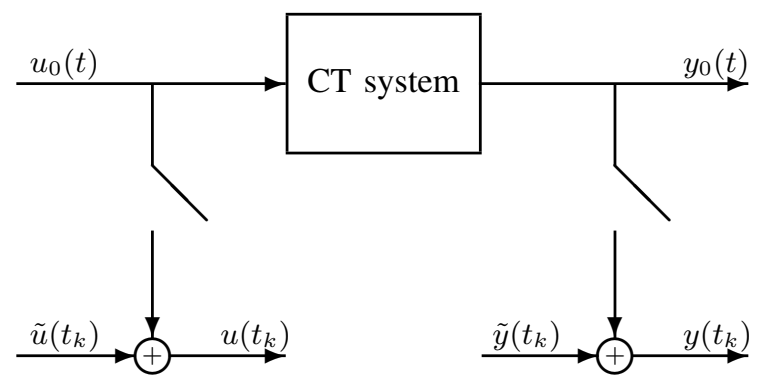

Fig. 1. CT system with noisy input/output data

Consider a CT linear time-invariant system represented in Figure 1. The noise-free input and output signals are related by

$$
y_{0}(t)=G_{0}(p) u_{0}(t)=\frac{B_{0}(p)}{A_{0}(p)} u_{0}(t)
$$

where $p$ is the differential operator and $G_{0}(p)$ is the transfer function of the true system. We assume that $u_{0}(t)$ and $y_{0}(t)$ are sampled at time-instants $\left\{t_{k}\right\}_{k=1}^{N}$. The sampled signals are both contaminated by discrete-time noise sequences, denoted as $\tilde{u}\left(t_{k}\right)$ and $\tilde{y}\left(t_{k}\right)$ respectively. The measured input and output signals are therefore given by

$$
\begin{aligned}
& u\left(t_{k}\right)=u_{0}\left(t_{k}\right)+\tilde{u}\left(t_{k}\right) \\
& y\left(t_{k}\right)=y_{0}\left(t_{k}\right)+\tilde{y}\left(t_{k}\right)
\end{aligned}
$$


The data-generating CT system is thus characterized by

$$
\mathcal{S}:\left\{\begin{array}{l}
y_{0}(t)=G_{0}(p) u_{0}(t)=\frac{B_{0}(p)}{A_{0}(p)} u_{0}(t) \\
u\left(t_{k}\right)=u_{0}\left(t_{k}\right)+\tilde{u}\left(t_{k}\right) \\
y\left(t_{k}\right)=y_{0}\left(t_{k}\right)+\tilde{y}\left(t_{k}\right)
\end{array}\right.
$$

It is then parameterized as follows

$$
\mathcal{G}:\left\{\begin{array}{l}
G(p, \boldsymbol{\theta})=B(p, \boldsymbol{\theta}) / A(p, \boldsymbol{\theta}) \\
A(p, \boldsymbol{\theta})=a_{0}+a_{1} p+\ldots+a_{n_{a}-1} p^{n_{a}-1}+p^{n_{a}} \\
B(p, \boldsymbol{\theta})=b_{0}+b_{1} p+\ldots+b_{n_{b}} p^{n_{b}}
\end{array}\right.
$$

with $\boldsymbol{\theta}^{T}=\left[\begin{array}{lllllll}a_{0} & \ldots & a_{n_{a}-1} & b_{0} & \ldots & b_{n_{b}}\end{array}\right]$ and $n_{a} \geqslant n_{b}$. In addition to the aforesaid conditions, we assume that

A1. $A_{0}(s) \neq 0$ for $\Re(s) \geq 0$ and $A_{0}(s), B_{0}(s)$ are coprime ( $s$ is the Laplace variable);

A2. the polynomial degrees $n_{a}$ and $n_{b}$ are a priori known;

A3. the noise-free signal $u_{0}\left(t_{k}\right)$ is a zero-mean stationary stochastic process such that its fourth-order cumulants are non-zero. Therefore, its probability density function cannot be Gaussian;

A4. $\tilde{u}\left(t_{k}\right)$ and $\tilde{y}\left(t_{k}\right)$ are stationary, zero-mean Gaussian random variables, independent of $u_{0}\left(t_{k}\right)$ and $y_{0}\left(t_{k}\right)$.

\section{REMARK 1}

Assumption Al aggregates the traditional assumptions of stability and observability of the system. We also suppose that the system (1) has been in operation for a time long enough so that the output $y_{0}$ is fourth-order wide-sense stationary, i.e. its moments of order up to four are invariant to any time shift.

Assumption A2 means that only the system belongs to the model class, a situation denoted as $G_{0} \in \mathcal{G}$ [12].

Assumptions A3 and A4 are linked to properties of higherorder statistics, and will be justified in the sequel.

Note that, except A4, there is no other assumption on the noises. They can thus be white or colored, and mutually correlated or not.

The identification problem can then be formulated as follows: given $N$ samples of noisy input/output data $\left\{u\left(t_{k}\right), y\left(t_{k}\right)\right\}_{k=1}^{N}$, directly estimate the continuous-time parameter vector $\boldsymbol{\theta}$.

\section{PROPERTIES OF FOURTH-ORDER CUMULANTS}

The identification technique developed in this paper is based on higher-order statistics (HOS) [5], [14], [10], [22]. The main definitions and properties used in the proposed approach are recalled in this section.

The fourth-order cumulant of a real-valued, zero-mean stationary random process $x\left(t_{k}\right)$ is defined as

$$
\begin{aligned}
& C_{x x x x}\left(\tau_{1}, \tau_{2}, \tau_{3}\right)=\operatorname{Cum}\left[x(t) x\left(t+\tau_{1}\right) x\left(t+\tau_{2}\right) x\left(t+\tau_{3}\right)\right] \\
&= E\left[x(t) x\left(t+\tau_{1}\right) x\left(t+\tau_{2}\right) x\left(t+\tau_{3}\right)\right] \\
&-E\left[x(t) x\left(t+\tau_{1}\right)\right] E\left[x\left(t+\tau_{2}\right) x\left(t+\tau_{3}\right)\right] \\
&-E\left[x(t) x\left(t+\tau_{2}\right)\right] E\left[x\left(t+\tau_{1}\right) x\left(t+\tau_{3}\right)\right] \\
&-E\left[x(t) x\left(t+\tau_{3}\right)\right] E\left[x\left(t+\tau_{1}\right) x\left(t+\tau_{2}\right)\right]
\end{aligned}
$$

The cumulants of order higher than two have many properties amongst which we only recall those used in the proposed estimation scheme.

Let $\boldsymbol{x}=\left[x\left(t_{1}\right), \ldots, x\left(t_{k}\right)\right]^{T}$ and $\boldsymbol{y}=\left[y\left(t_{1}\right), \ldots, y\left(t_{k}\right)\right]^{T}$ be two random vectors.

P1. Multilinearity: if $\boldsymbol{x}$ and $\boldsymbol{y}$ are linearly linked up by $\boldsymbol{y}=$ $\boldsymbol{M x}$, where $\boldsymbol{M}$ is any matrix of appropriate size, then the cumulants of $\boldsymbol{y}$ are linear functions of each of the components $M_{i j}$. For instance

$$
\begin{aligned}
& \operatorname{Cum}\left[y\left(t_{i}\right), y\left(t_{j}\right), y\left(t_{k}\right), y\left(t_{l}\right)\right] \\
= & \sum_{a, b, c, d} M_{i a} M_{j b} M_{k c} M_{l d} \operatorname{Cum}\left[x\left(t_{a}\right), x\left(t_{b}\right), x\left(t_{c}\right), x\left(t_{d}\right)\right]
\end{aligned}
$$

P2. Additivity: if $\boldsymbol{x}$ and $\boldsymbol{y}$ are independent, the cumulant of their sum equals the sum of their cumulants

$$
\begin{aligned}
& \operatorname{Cum}\left[x\left(t_{1}\right)+y\left(t_{1}\right), \ldots, x\left(t_{n}\right)+y\left(t_{n}\right)\right] \\
= & \operatorname{Cum}\left[x\left(t_{1}\right), \ldots, x\left(t_{n}\right)\right]+\operatorname{Cum}\left[y\left(t_{1}\right), \ldots, y\left(t_{n}\right)\right]
\end{aligned}
$$

P3. The fourth-order cumulant of a random variable with a Gaussian distribution is equal to zero.

Now the relevance of assumptions A3 and A4 is pointed out: if the fourth-order cumulant of the measured input signal (2) is considered, using properties P2 and P3 yields ${ }^{1}$

$$
\begin{aligned}
C_{\text {uиuu }}(\boldsymbol{\tau}) & =C_{u_{0} u_{0} u_{0} u_{0}}(\boldsymbol{\tau})+C_{\tilde{u} \tilde{u} \tilde{u} \tilde{u}}(\boldsymbol{\tau}) \\
& =C_{u_{0} u_{0} u_{0} u_{0}}(\boldsymbol{\tau})
\end{aligned}
$$

In the same manner this result is obviously true for the fourth-order cumulant of any combination of input/output signals. The use of fourth-order cumulants therefore allows to get naturally rid of the (possibly colored) measurement noise contaminating the input/output data, under assumptions A3-A4.

\section{HOS-BASED METHODS FOR CT EIV MODELS}

\section{PROPOSITION 1}

The fourth-order cross-cumulant between the measured input/output signals satisfies

$$
C_{\text {uуuи }}(\boldsymbol{\tau})=\frac{B(p, \boldsymbol{\theta})}{A(p, \boldsymbol{\theta})} C_{\text {uиuи }}(\boldsymbol{\tau})
$$

where the differential operator $p$ stands for $\frac{\partial}{\partial \tau_{1}}$.

This result is the starting point of the identification methods: the differential equation of the system is also satisfied by the fourth-order cumulants. If the input/ouput signals are contaminated by Gaussian noises, then (9) only involves noise-free terms. Thus theoretically (i.e. with an infinite data set) any consistent parametric identification method applied to (9) will give the exact parameter vector. In the sequel two such possibilities are presented.

Several problems arise though, mostly due to the fact that the cumulants have to be estimated from a finite number of the data.

\footnotetext{
${ }^{1}$ For ease of notation $\boldsymbol{\tau}=\left[\begin{array}{lll}\tau_{1} & \tau_{2} & \tau_{3}\end{array}\right]^{T}$ is used in the sequel.
} 


\section{A. Estimation of the fourth-order cumulants}

Given $N$ samples of input/output data $\left\{u\left(t_{k}\right), y\left(t_{k}\right)\right\}_{k=1}^{N}$ the fourth-order cumulants have to be estimated. A natural estimator from $\mathrm{N}$ samples is obtained by using sample averages instead of mathematical expectations:

$$
\begin{gathered}
\widehat{C}_{x x x x}(\boldsymbol{\tau})=\frac{1}{N} \sum_{k=1}^{N} x\left(t_{k}\right) x\left(t_{k}+\tau_{1}\right) x\left(t_{k}+\tau_{2}\right) x\left(t_{k}+\tau_{3}\right) \\
-\frac{1}{N^{2}} \sum_{k=1}^{N} x\left(t_{k}\right) x\left(t_{k}+\tau_{1}\right) \sum_{k=1}^{N} x\left(t_{k}+\tau_{2}\right) x\left(t_{k}+\tau_{3}\right) \\
-\frac{1}{N^{2}} \sum_{k=1}^{N} x\left(t_{k}\right) x\left(t_{k}+\tau_{2}\right) \sum_{k=1}^{N} x\left(t_{k}+\tau_{1}\right) x\left(t_{k}+\tau_{3}\right) \\
-\frac{1}{N^{2}} \sum_{k=1}^{N} x\left(t_{k}\right) x\left(t_{k}+\tau_{3}\right) \sum_{k=1}^{N} x\left(t_{k}+\tau_{1}\right) x\left(t_{k}+\tau_{2}\right)
\end{gathered}
$$

Some simple calculations show that this estimator is asymptotically unbiased, but biased for finite $N$. Thus with a large number of samples (10) is an approximatively unbiased estimator. However, when only a small data set is available, the bias cannot be neglected anymore. For such situations, it becomes crucial to use an unbiased estimator, as the $k$ statistic given by [10], [5], [22]:

$$
\begin{gathered}
\widehat{C}_{x x x x}(\boldsymbol{\tau})=\frac{1}{(N-1)(N-2)(N-3)}\{ \\
N(N+1) \sum_{k=1}^{N} x\left(t_{k}\right) x\left(t_{k}+\tau_{1}\right) x\left(t_{k}+\tau_{2}\right) x\left(t_{k}+\tau_{3}\right) \\
-3(N-1) \sum_{k=1}^{N} x\left(t_{k}\right) x\left(t_{k}+\tau_{1}\right) \sum_{k=1}^{N} x\left(t_{k}+\tau_{2}\right) x\left(t_{k}+\tau_{3}\right) \\
-3(N-1) \sum_{k=1}^{N} x\left(t_{k}\right) x\left(t_{k}+\tau_{2}\right) \sum_{k=1}^{N} x\left(t_{k}+\tau_{1}\right) x\left(t_{k}+\tau_{3}\right) \\
\left.-3(N-1) \sum_{k=1}^{N} x\left(t_{k}\right) x\left(t_{k}+\tau_{3}\right) \sum_{k=1}^{N} x\left(t_{k}+\tau_{1}\right) x\left(t_{k}+\tau_{2}\right)\right\}
\end{gathered}
$$

From a finite number of data, by using (11), estimates of the fourth-order cumulants are calculated. Then (9) can be rewritten as:

$$
\begin{aligned}
\widehat{C}_{\text {uуиu }}(\boldsymbol{\tau}) & =\frac{B(p, \boldsymbol{\theta})}{A(p, \boldsymbol{\theta})} \widehat{C}_{\text {ииии }}(\boldsymbol{\tau})+\varepsilon(\boldsymbol{\tau}, \boldsymbol{\theta}) \\
\varepsilon(\boldsymbol{\tau}, \boldsymbol{\theta}) & =\frac{B(p, \boldsymbol{\theta})}{A(p, \boldsymbol{\theta})} \tilde{C}_{\text {ииии }}(\boldsymbol{\tau})-\tilde{C}_{\text {uуии }}(\boldsymbol{\tau})
\end{aligned}
$$

where $\tilde{C}_{x x x x}=\widehat{C}_{x x x x}+\tilde{C}_{x x x x}$ denotes the estimation error of $C_{x x x x}$, that is:

$$
C_{x x x x}=\widehat{C}_{x x x x}+\tilde{C}_{x x x x}
$$

Since the cumulant estimates (11) are unbiased and consistent, it holds that:

$$
\lim _{N \rightarrow \infty} \varepsilon(\boldsymbol{\tau}, \boldsymbol{\theta})=0 \quad \text { w.p. } 1
$$

\section{B. Cumulant-based LS approach}

Equation (12) can be rewritten under a linear regression form

$$
\widehat{C}_{\text {uyuu }}^{\left(n_{a}\right)}(\boldsymbol{\tau})=\widehat{\boldsymbol{\Phi}}^{T}(\boldsymbol{\tau}) \boldsymbol{\theta}+A(p, \boldsymbol{\theta}) \varepsilon(\boldsymbol{\tau}, \boldsymbol{\theta})
$$

where

$$
\widehat{C}_{u y u u}^{(j)}(\boldsymbol{\tau}) \triangleq \frac{\partial^{j}}{\partial \tau_{1}^{j}} \widehat{C}_{\text {uyuu }}(\boldsymbol{\tau})
$$

and the regression vector is given by

$$
\begin{aligned}
\widehat{\boldsymbol{\Phi}}^{T}(\boldsymbol{\tau})=\left[-\widehat{C}_{\text {uуuи }}^{(0)}(\boldsymbol{\tau})\right. & \ldots-\widehat{C}_{\text {uyuu }}^{\left(n_{a}-1\right)}(\boldsymbol{\tau}) \\
& \left.\widehat{C}_{\text {uuuu }}^{(0)}(\boldsymbol{\tau}) \ldots \widehat{C}_{\text {uuuu }}^{\left(n_{b}\right)}(\boldsymbol{\tau})\right]
\end{aligned}
$$

Define then the error function

$$
\begin{aligned}
e(\boldsymbol{\tau}, \boldsymbol{\theta}) & =\widehat{C}_{\text {uуuи }}^{\left(n_{a}\right)}(\boldsymbol{\tau})-\widehat{\boldsymbol{\Phi}}^{T}(\boldsymbol{\tau}) \boldsymbol{\theta} \\
& =B(p, \boldsymbol{\theta}) \tilde{C}_{\text {uиuu }}(\boldsymbol{\tau})-A(p, \boldsymbol{\theta}) \tilde{C}_{\text {uyuu }}(\boldsymbol{\tau})
\end{aligned}
$$

Minimizing the following cost function with respect to $\theta$

$$
V\left(\tau_{2}, \tau_{3}, \boldsymbol{\theta}, M\right)=\frac{1}{M} \sum_{\tau_{1}=0}^{M-1} \frac{1}{2} e^{2}(\boldsymbol{\tau}, \boldsymbol{\theta})
$$

leads to the focls estimator (Fourth-Order Cumulants based Least Squares algorithm), given by

$$
\begin{aligned}
\widehat{\boldsymbol{\theta}}_{\text {focls }}\left(\tau_{2}, \tau_{3}, M\right)= & {\left[\frac{1}{M} \sum_{\tau_{1}=0}^{M-1} \widehat{\boldsymbol{\Phi}}(\boldsymbol{\tau}) \widehat{\boldsymbol{\Phi}}^{T}(\boldsymbol{\tau})\right]^{-1} } \\
& {\left[\frac{1}{M} \sum_{\tau_{1}=0}^{M-1} \widehat{\boldsymbol{\Phi}}(\boldsymbol{\tau}) \widehat{C}_{\text {uyuu }}^{\left(n_{a}\right)}(\boldsymbol{\tau})\right] }
\end{aligned}
$$

\section{REMARK 2}

- The role of the user parameter $M$ is to prevent the use of cumulants with large time-lags: since the cumulants are estimated using sample averages, when the timelags increase the estimates will be calculated with less data, thus becoming less reliable. This phenomenon is underlined in Section V-B.

- By definition the fourth-order cumulants of a stationary signal are three-dimensional, in the sense that they depend on three variables (here $\tau_{1}, \tau_{2}$ and $\tau_{3}$ ). From the available data we can thus derive a cube of length $N$ constituted of the third-order cumulants calculated at different points. It has been mentioned above that it is better to consider a cube of length $M$ containing only reliable estimates of the cumulants. But it is yet unclear if all that cube should be used, or only a part of it (called 'slice'). Estimation of numerous cumulants and then calculation of the parameter estimate can indeed be time-consuming. These questions are strongly linked to the estimation of the cumulants, and a thorough analysis is beyond the scope of this paper. To the authors' knowledge, there is no definitive rule telling what slices to use. In this paper a very simple slice has been considered: the vector obtained with the setting $\tau_{2}=\tau_{3}=0$ and $0 \leqslant \tau_{1} \leqslant M$. 


\section{Cumulant-based iterative LS approach}

Equations (12)-(13) define an output error given by

$$
\begin{aligned}
\varepsilon(\boldsymbol{\tau}, \boldsymbol{\theta}) & =\widehat{C}_{\text {uуuu }}(\boldsymbol{\tau})-\frac{B(p, \boldsymbol{\theta})}{A(p, \boldsymbol{\theta})} \widehat{C}_{\text {uиuu }}(\boldsymbol{\tau}) \\
& =\frac{B(p, \boldsymbol{\theta})}{A(p, \boldsymbol{\theta})} \tilde{C}_{\text {uиuu }}(\boldsymbol{\tau})-\tilde{C}_{\text {uyuu }}(\boldsymbol{\tau})
\end{aligned}
$$

This error is nonlinear in the parameters. To avoid the recourse to nonlinear optimization methods, the proposed second approach is based on an iterative LS procedure, as initially suggested for discrete-time model identification [4], [3].

The output error (23) can be rewritten as

$$
\begin{aligned}
\varepsilon(\boldsymbol{\tau}, \boldsymbol{\theta}) & =\frac{1}{A(p, \boldsymbol{\theta})}\left(A(p, \boldsymbol{\theta}) \widehat{C}_{\text {uyuu }}(\boldsymbol{\tau})-B(p, \boldsymbol{\theta}) \widehat{C}_{\text {uuuu }}(\boldsymbol{\tau})\right) \\
& =A(p, \boldsymbol{\theta}) \widehat{C}_{u y u u, f}(\boldsymbol{\tau})-B(p, \boldsymbol{\theta}) \widehat{C}_{u u u u, f}(\boldsymbol{\tau})
\end{aligned}
$$

where $\widehat{C}_{\text {uuuu,f }}$ and $\widehat{C}_{u y u u, f}$ denote the cumulants filtered by $1 / A(p, \boldsymbol{\theta})$. An equation error is thus obtained, linear in the parameters, which can be estimated by the least squares method.

Since $A(p, \boldsymbol{\theta})$ is unknown, the idea is to proceed in an iterative fashion to transform the equation error (25) into the output error (23). Let $\widehat{\boldsymbol{\theta}}^{i}$ be the estimate of $\boldsymbol{\theta}$ at the $i$ th iteration. At each iteration, $\widehat{\boldsymbol{\theta}}^{i+1}$ is given by a least squares estimate, using the cumulants filtered by $1 / A\left(p, \widehat{\boldsymbol{\theta}}^{i}\right)$. Insomuch as the parameters converge to a constant value, we have

$$
\frac{A\left(p, \widehat{\boldsymbol{\theta}}^{i+1}\right)}{A\left(p, \widehat{\boldsymbol{\theta}}^{i}\right)} \longrightarrow 1 \text { and } \frac{B\left(p, \widehat{\boldsymbol{\theta}}^{i+1}\right)}{A\left(p, \widehat{\boldsymbol{\theta}}^{i}\right)} \longrightarrow \frac{B\left(p, \widehat{\boldsymbol{\theta}}^{i+1}\right)}{A\left(p, \widehat{\boldsymbol{\theta}}^{i+1}\right)}
$$

Hence, the equation error (25) tends toward the output error (23). The convergence of this type of iterative algorithm is very fast: typically a few iterations are enough [26].

The equation error (25) can be rewritten as a linear regression

$$
\varepsilon(\boldsymbol{\tau}, \boldsymbol{\theta})=\widehat{C}_{\text {uyuu }, f}^{\left(n_{a}\right)}(\boldsymbol{\tau})-\widehat{\boldsymbol{\Phi}}_{f}^{T}(\boldsymbol{\tau}) \boldsymbol{\theta}
$$

where the regression vector is

$$
\begin{array}{r}
\widehat{\boldsymbol{\Phi}}_{f}^{T}(\boldsymbol{\tau})=\left[\begin{array}{lll}
-\widehat{C}_{\text {uyuu }, f}^{(0)}(\boldsymbol{\tau}) & \ldots & -\widehat{C}_{\text {uyuu }, f}^{\left(n_{a}-1\right)}(\boldsymbol{\tau}) \\
& \widehat{C}_{\text {uuuu }, f}^{(0)}(\boldsymbol{\tau}) \ldots \widehat{C}_{\text {uuuu }, f}^{\left(n_{b}\right)}(\boldsymbol{\tau})
\end{array}\right]
\end{array}
$$

The focils estimator (Fourth-Order Cumulants based Iterative Least Squares algorithm) is given by

$$
\begin{aligned}
\widehat{\boldsymbol{\theta}}_{\text {focils }}\left(\tau_{2}, \tau_{3}, M\right)= & {\left[\frac{1}{M} \sum_{\tau_{1}=0}^{M-1} \widehat{\boldsymbol{\Phi}}_{f}(\boldsymbol{\tau}) \widehat{\boldsymbol{\Phi}}_{f}^{T}(\boldsymbol{\tau})\right]^{-1} } \\
& {\left[\frac{1}{M} \sum_{\tau_{1}=0}^{M-1} \widehat{\boldsymbol{\Phi}}_{f}(\boldsymbol{\tau}) \widehat{C}_{\text {uyuu }, f}^{\left(n_{a}\right)}(\boldsymbol{\tau})\right] }
\end{aligned}
$$

\section{REMARK 3}

This iterative method is greatly inspired by the algorithm of [20]. There is however a major difference: the SteiglitzMcBride algorithm uses the iterative LS estimator on measured I/O data rather than on the cumulants, being therefore applicable under quite restrictive assumptions (white measurement noise on the output) as it is pointed out in [21]. The proposed method uses that procedure on the fourthorder cumulants, and since they are insensitive to (white or colored) Gaussian mesurement noises, the same restrictions do not apply.

\section{Handling of the cumulant time-derivatives: the SVF ap- proach}

The time-derivatives of the cumulants are needed to build up the regression vectors (18), (27). These can be obtained e.g. by using the traditional state variable filtering (SVF) approach, whose basics are quickly recalled in the sequel (see [27], [28], [9] for further details).

Let $n_{\max }$ be the maximum of $n_{a}$ and $n_{b}$. The SVF approach allows to reconstruct the time-derivatives (up to an order $n_{\max }$ ) of a signal by passing it into the following filter bank

$$
F_{n}(p)=p^{n}\left(\frac{\lambda}{p+\lambda}\right)^{n_{\max }}, \quad 0 \leqslant n \leqslant n_{\max }
$$

where $\lambda$ is a user parameter accounting for the filter cut-off frequency. Intuitively, it can be chosen in order to emphasize the frequency band of interest and generally, it should be chosen close to, or larger than the bandwidth of the system to be identified.

The filter bank outputs provide the time-derivatives of the signal in the bandwidth of interest

$$
\begin{aligned}
x_{f}^{(n)}(t) & =F_{n}(p) x(t) \\
& =\left[f_{n} \star x\right](t)
\end{aligned}
$$

where $\star$ stands for the convolution operator and $f_{n}(t)$ denotes the impulse response of the linear time-invariant filter $F_{n}(s)$

$$
f_{n}(t)=\mathcal{L}^{-1}\left[F_{n}(s)\right]
$$

with $\mathcal{L}$ symbolizing the Laplace transform.

The time-derivatives of the cumulants are now computed using this SVF approach. As a result of their multilinearity property, a formula for cumulant filtering can be derived [10], [15]

$$
\begin{aligned}
& C_{x x x x, f}^{(n)}(\boldsymbol{\tau})=F_{n}(p) C_{x x x x}(\boldsymbol{\tau}) \\
& \quad=\int_{\mathbb{R}} f_{n}(\tau) C_{x x x x}\left(\tau_{1}-\tau, \tau_{2}, \tau_{3}\right) d \tau \\
& \quad=\int_{\mathbb{R}} f_{n}(\tau) E\left[x(t) x\left(t+\tau_{1}-\tau\right) x\left(t+\tau_{2}\right) x\left(t+\tau_{3}\right)\right] d \tau \\
& \quad=E\left[x(t)\left(\int_{\mathbb{R}} f_{n}(\tau) x\left(t+\tau_{1}-\tau\right) d \tau\right) x\left(t+\tau_{2}\right) x\left(t+\tau_{3}\right)\right] \\
& \quad=E\left[x(t) x_{f}^{(n)}\left(t+\tau_{1}\right) x\left(t+\tau_{2}\right) x\left(t+\tau_{3}\right)\right]
\end{aligned}
$$


We thus obtain

$$
C_{x x x x, f}^{(n)}(\boldsymbol{\tau})=C_{x x_{f}^{(n)} x x}(\boldsymbol{\tau})
$$

Consequently, the use of the SVF approach, that is the application on both sides of equation (9) of the filters $\left\{F_{n}(p)\right\}_{n=0}^{n_{\max }}$, allows to transfer the cumulant time-derivative estimation problem to an input/output signal time-derivative estimation problem, which constitutes a well-known task in continuous-time model identification [9].

In the focls algorithm, the basic filter bank (29) is applied to the input/output signals. Their time-derivatives in the bandwidth of interest are therefore obtained, from which the cumulants time-derivatives are computed. In the focils algorithm however, the basic filter bank (29) is applied to the input/output signals only at the initialization stage (or equivalently: the focils algorithm is initialized with the focls estimate). In the subsequent iterations, as an estimate $\widehat{\boldsymbol{\theta}}_{\text {focils }}^{i}\left(\tau_{2}, \tau_{3}, M\right)$ is available, the following filter bank is used

$$
F_{n}(p)=\frac{p^{n}}{A\left(p, \widehat{\boldsymbol{\theta}}_{\text {focils }}^{i}\left(\tau_{2}, \tau_{3}, M\right)\right)}, \quad 0 \leqslant n \leqslant n_{\max }
$$

Note that this filter bank allows to realize both the filtering by $1 / A\left(p, \widehat{\boldsymbol{\theta}}_{\text {focils }}^{i}\left(\tau_{2}, \tau_{3}, M\right)\right)$ and the differentiation in one step only.

\section{NumERICAL EXAMPLE}

The following second-order system is considered throughout the example (coming from [13])

$$
G_{0}(p)=\frac{p-1}{p^{2}+2 p+1}
$$

The noise-free input signal $u_{0}$ is chosen as a multi-sine signal:

$$
u_{0}(t)=\sin (t)+\sin (1.9 t)+\sin (2.1 t)+\sin (2.3 t)
$$

The input/ouput signals are sampled uniformly with a sampling interval of $0.05 \mathrm{~s}$. The performance of the algorithms are assessed with the help of Monte Carlo simulations of $n_{m c}=50$ runs. The noise-free and noisy signals are plotted on Figure 2.

\section{A. Performance analysis}

We first focus on the performance of the proposed algorithms and compare them with the performance of the method of [13], referenced to as eivsvf.

1) White noises on $1 / O$ : In this part the noises contaminating the input/output signals are defined as Gaussian white noises, whose variances are adjusted so that the signal-to-noise ratio (SNR) is equal to $5 \mathrm{~dB}$ on both input and output, with

$$
\mathrm{SNR}=10 \log _{10}\left(\frac{P_{x_{0}}}{P_{\tilde{x}}}\right)
$$

where $P_{\tilde{x}}$ represents the average power of the zero-mean additive noise while $P_{x_{0}}$ denotes the average power of the noise-free signal fluctuations.

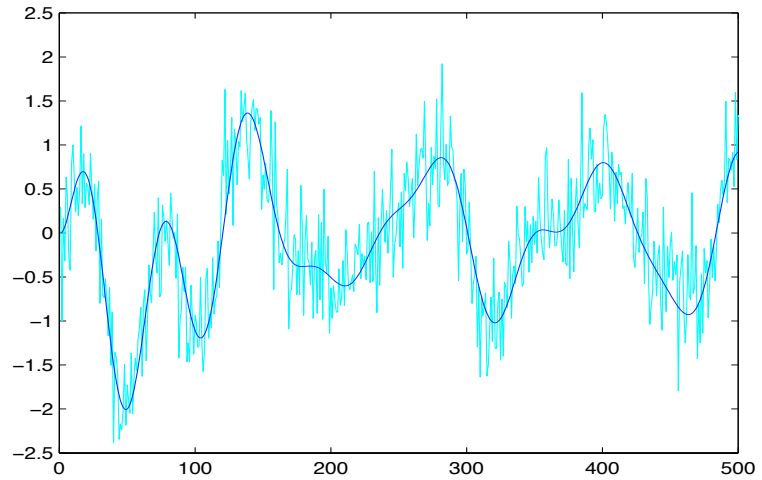

(a) Output signal

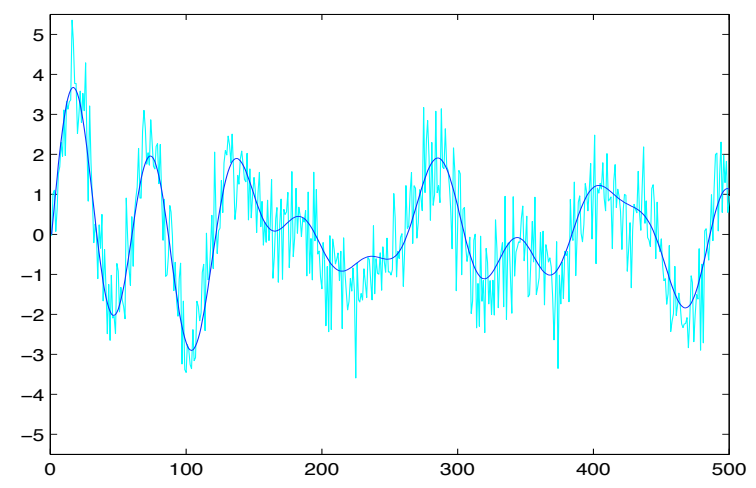

(b) Input signal

Fig. 2. Part of the noise-free and noisy input/output signals.

Table I and Table II contain the mean and standard deviation of the estimates for $N=1000$ and $N=5000$ respectively as well as the normalized root mean square error, defined as

$$
\mathrm{NRMSE} \triangleq \sqrt{\frac{1}{n_{m c}} \sum_{j=1}^{n_{m c}} \frac{\left\|\widehat{\boldsymbol{\theta}}_{j}-\boldsymbol{\theta}\right\|^{2}}{\|\boldsymbol{\theta}\|^{2}}}
$$

where $\|$.$\| is the Euclidian norm and \widehat{\boldsymbol{\theta}}_{j}$ is the parameter vector estimate obtained at the $j$ th run of the Monte Carlo simulation. The other entries of Table 1 are $\lambda, M$ and \#it, respectively the cut-off frequency of the SVF, the user parameter of the proposed methods and the mean of the iteration number needed to achieve convergence.

The Bode diagrams of the true and estimated models are plotted in Figure 3.

2) Colored noise on output: The noises contaminating the input/output signals are defined as

$$
\tilde{u}\left(t_{k}\right)=e_{1}\left(t_{k}\right) \quad \tilde{y}\left(t_{k}\right)=\frac{1+0.7 q^{-1}}{1-0.7 q^{-1}} e_{2}\left(t_{k}\right)
$$

where $q^{-1}$ is the backward shift operator, i.e. $q^{-1} x\left(t_{k}\right)=$ $x\left(t_{k-1}\right)$ and $e_{1}\left(t_{k}\right), e_{2}\left(t_{k}\right)$ are zero-mean Gaussian white noises. The variances of $e_{i}\left(t_{k}\right)$ are then adjusted so that the $\mathrm{SNR}$ is equal to $5 \mathrm{~dB}$ on both input and output. 


\begin{tabular}{|c|c|c|c|c|c|c|c|c|}
\hline & $\lambda$ & $M$ & $a_{1}=2$ & $a_{0}=1$ & $b_{1}=1$ & $b_{0}=-1$ & NRMSE & \#it \\
\hline eivsvf & - & - & $\begin{array}{l}2.135 \\
\pm 0.186 \\
\end{array}$ & $\begin{array}{l}0.998 \\
\pm 0.127 \\
\end{array}$ & $\begin{array}{l}1.012 \\
\pm 0.088 \\
\end{array}$ & $\begin{array}{c}-1.091 \\
\pm 0.098\end{array}$ & $10.8 \%$ & - \\
\hline \multirow{2}{*}{ focls } & \multirow{2}{*}{3} & 110 & $\begin{array}{l}2.005 \\
\pm 0.171\end{array}$ & $\begin{array}{l}1.030 \\
\pm 0.157\end{array}$ & $\begin{array}{l}0.990 \\
\pm 0.082\end{array}$ & $\begin{array}{l}-0.978 \\
\pm 0.100\end{array}$ & $9.5 \%$ & - \\
\hline & & 160 & $\begin{array}{c}1.983 \\
\pm 0.175\end{array}$ & $\begin{array}{c}1.026 \\
\pm 0.142 \\
\end{array}$ & $\begin{array}{c}0.983 \\
\pm 0.080 \\
\end{array}$ & $\begin{array}{l}-0.981 \\
\pm 0.096\end{array}$ & $9.2 \%$ & - \\
\hline \multirow{2}{*}{ focils } & \multirow{2}{*}{-} & 110 & $\begin{array}{l}2.025 \\
\pm 0.168\end{array}$ & $\begin{array}{c}1.039 \\
\pm 0.131\end{array}$ & $\begin{array}{c}0.997 \\
\pm 0.079\end{array}$ & $\begin{array}{l}-0.967 \\
\pm 0.088\end{array}$ & $8.9 \%$ & 3 \\
\hline & & 160 & $\begin{array}{l}2.012 \\
\pm 0.168 \\
\end{array}$ & $\begin{array}{c}1.031 \\
\pm 0.133\end{array}$ & $\begin{array}{c}0.997 \\
\pm 0.080 \\
\end{array}$ & $\begin{array}{l}-0.975 \\
\pm 0.095\end{array}$ & $8.9 \%$ & 2.9 \\
\hline
\end{tabular}

TABLE I

MEAN AND STANDARD DEVIATION OF THE PARAMETER ESTIMATES (WHITE GAUSSIAN NOISES ON I/O, $N=1000$ ).

\begin{tabular}{|c|c|c|c|c|c|c|c|c|}
\hline & $\lambda$ & $M$ & $a_{1}=2$ & $a_{0}=1$ & $b_{1}=1$ & $b_{0}=-1$ & NRMSE & \#it \\
\hline eivsvf & - & - & $\begin{array}{l}2.131 \\
\pm 0.077\end{array}$ & $\begin{array}{l}0.978 \\
\pm 0.071\end{array}$ & $\begin{array}{l}1.021 \\
\pm 0.041\end{array}$ & $\begin{array}{l}-1.098 \\
\pm 0.046\end{array}$ & $7.3 \%$ & - \\
\hline \multirow{2}{*}{ focls } & \multirow{2}{*}{3} & & $\begin{array}{l}1.997 \\
\pm 0.072\end{array}$ & $\begin{array}{l}1.035 \\
\pm 0.082\end{array}$ & $\begin{array}{l}0.985 \\
\pm 0.036\end{array}$ & $\begin{array}{l}-0.968 \\
\pm 0.049\end{array}$ & $4.7 \%$ & - \\
\hline & & 160 & $\begin{array}{c}1.987 \\
\pm 0.071\end{array}$ & $\begin{array}{l}1.018 \\
\pm 0.071\end{array}$ & $\begin{array}{c}0.985 \\
\pm 0.034 \\
\end{array}$ & $\begin{array}{l}-0.977 \\
\pm 0.045\end{array}$ & $4.2 \%$ & - \\
\hline \multirow{2}{*}{ focils } & \multirow{2}{*}{-} & 110 & $\begin{array}{l}2.015 \\
\pm 0.072\end{array}$ & $\begin{array}{l}1.037 \\
\pm 0.075\end{array}$ & $\begin{array}{l}0.995 \\
\pm 0.035\end{array}$ & $\begin{array}{l}-0.971 \\
\pm 0.046\end{array}$ & $4.6 \%$ & 2.6 \\
\hline & & 160 & $\begin{array}{l}2.011 \\
\pm 0.070\end{array}$ & $\begin{array}{l}1.019 \\
\pm 0.072\end{array}$ & $\begin{array}{c}0.997 \\
\pm 0.035 \\
\end{array}$ & $\begin{array}{l}-0.974 \\
\pm 0.047\end{array}$ & $4.2 \%$ & 2.3 \\
\hline
\end{tabular}

TABLE II

MEAN AND STANDARD DEVIATION OF THE PARAMETER ESTIMATES (WHITE GAUSSIAN NOISES ON I/O, $N=5000$ ).

\begin{tabular}{|c|c|c|c|c|c|c|c|c|}
\hline & $\lambda$ & $M$ & $a_{1}=2$ & $a_{0}=1$ & $b_{1}=1$ & $b_{0}=-1$ & NRMSE & \#it \\
\hline eivsvf & - & - & $\begin{array}{l}2.129 \\
\pm 0.135\end{array}$ & $\begin{array}{l}1.014 \\
\pm 0.129\end{array}$ & $\begin{array}{l}1.342 \\
\pm 0.088\end{array}$ & $\begin{array}{l}-1.062 \\
\pm 0.088\end{array}$ & $15.3 \%$ & - \\
\hline \multirow{2}{*}{ focls } & \multirow{2}{*}{3} & 110 & $\begin{array}{c}1.970 \\
\pm 0.138\end{array}$ & $\begin{array}{c}1.043 \\
\pm 0.129\end{array}$ & $\begin{array}{l}0.972 \\
\pm 0.070\end{array}$ & $\begin{array}{l}-0.961 \\
\pm 0.089\end{array}$ & $8.2 \%$ & - \\
\hline & & 160 & $\begin{array}{c}1.955 \\
\pm 0.134\end{array}$ & $\begin{array}{c}1.029 \\
\pm 0.111\end{array}$ & $\begin{array}{c}0.971 \\
\pm 0.067\end{array}$ & $\begin{array}{l}-0.971 \\
\pm 0.084\end{array}$ & $7.6 \%$ & - \\
\hline \multirow{2}{*}{ focils } & \multirow{2}{*}{ - } & 110 & $\begin{array}{l}1.987 \\
\pm 0.137\end{array}$ & $\begin{array}{l}1.047 \\
\pm 0.117\end{array}$ & $\begin{array}{l}0.980 \\
\pm 0.067\end{array}$ & $\begin{array}{l}-0.953 \\
\pm 0.083\end{array}$ & $7.8 \%$ & 2.7 \\
\hline & & 160 & $\begin{array}{c}1.980 \\
\pm 0.134 \\
\end{array}$ & $\begin{array}{l}1.028 \\
\pm 0.115\end{array}$ & $\begin{array}{c}0.983 \\
\pm 0.067 \\
\end{array}$ & $\begin{array}{l}-0.971 \\
\pm 0.085\end{array}$ & $7.5 \%$ & 2.6 \\
\hline
\end{tabular}

TABLE III

MEAN AND STANDARD DEVIATION OF THE PARAMETER ESTIMATES (White/COLORED GAUSSIAN NOISES ON I/O, $N=5000$ ).

3) Discussion: In the case where the noises are both white, the three algorithms lead to good results. The proposed algorithms have a smaller bias than those stemming from the eivsvf method, whereas the variances are comparable. The NRMSE is thus slightly better for focls and focils. When the number of sample is large $(N=5000)$ the difference become more noticeable: the estimates of the fourth-order cumulants become more accurate, leading to improved performance of the proposed algorithms.

When the output measurement noise is colored, the eivsvf performance logically deteriorates, since it has not been developed to handle this case. The proposed algorithms still give good results, showing their robustness to Gaussian noises, whether they are colored or white.

\section{B. Influence of the user parameter $M$}

To study the influence of the user parameter $M$ on the quality of the estimates, the proposed algorithms have been applied for different values of $M$. The normalized root mean square

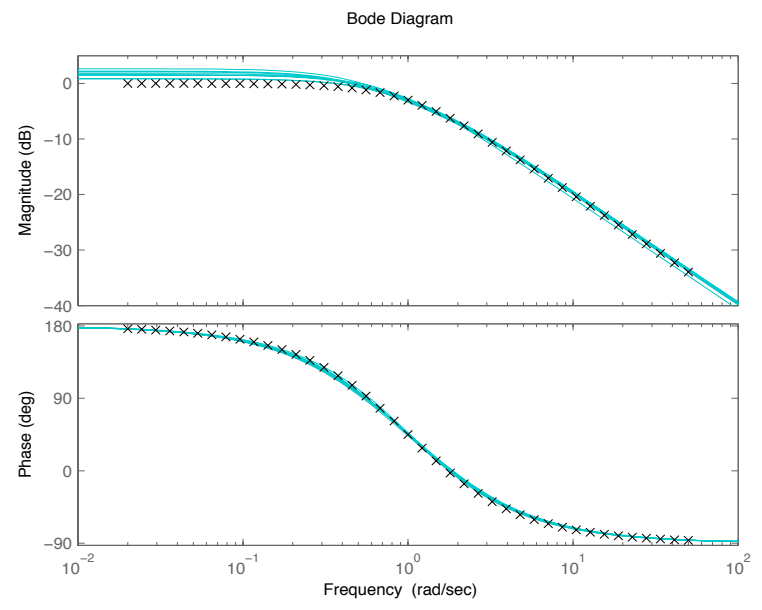

(a) eivsvf algorithm

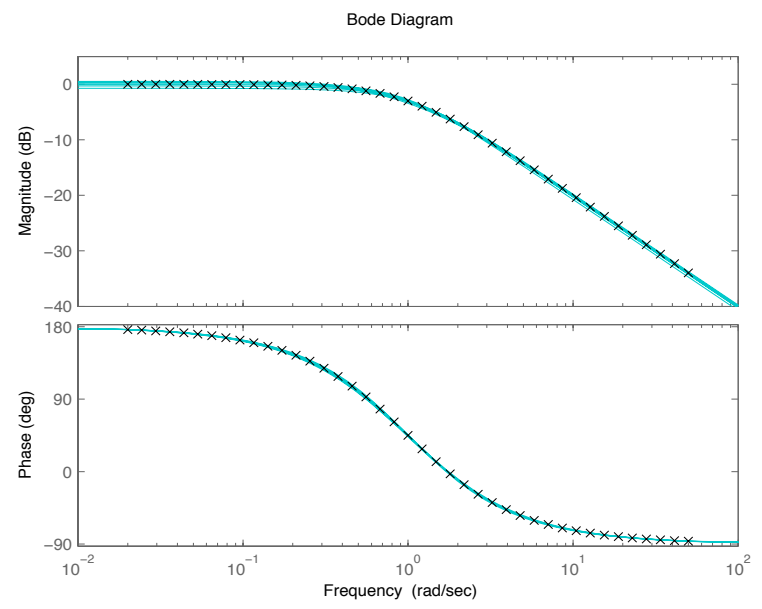

(b) focls algorithm

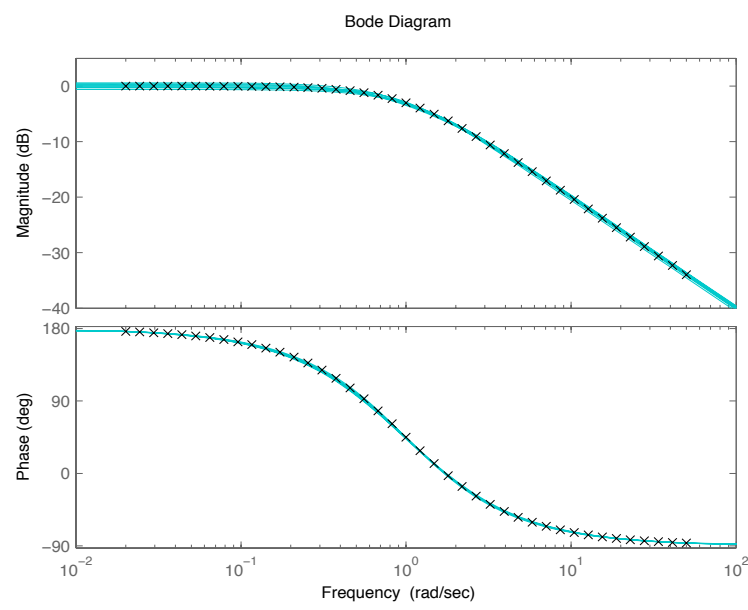

(c) focils algorithm

Fig. 3. Bode diagrams of the true (' $x$ ') and estimated models (white Gaussian noises $N=5000, M=160, \mathrm{SNR}=5 \mathrm{~dB}$ ).

error is plotted as a function of $M$ for $N=1000$ and for $N=5000$ in Figure 4.

It can be seen that the proposed approaches are not too 
sensitive to their user parameter, as long as it is not chosen too small, or too close to the data length. Indeed, the increase of the NRMSE in Figure 4-(a) is explained by the value of $M$ relatively to $N$ : the cumulants estimates are calculated using not enough data to be accurate, resulting in large errors in the parameter vector estimate.

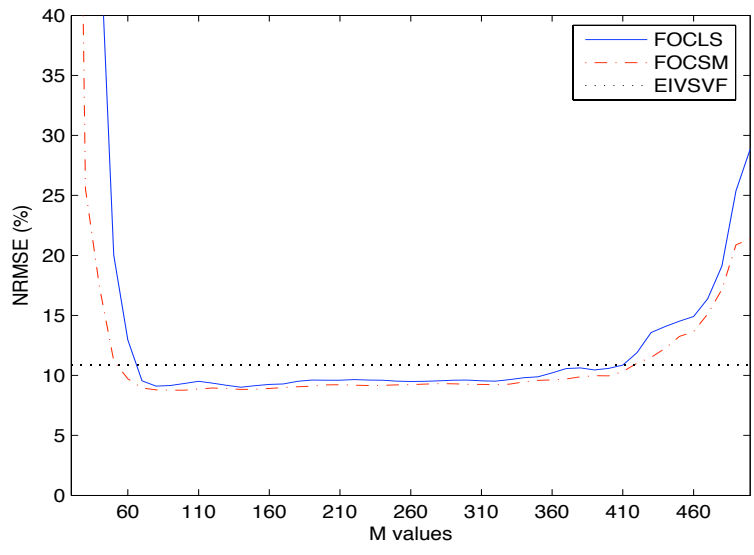

(a) White Gaussian noises, $N=1000$

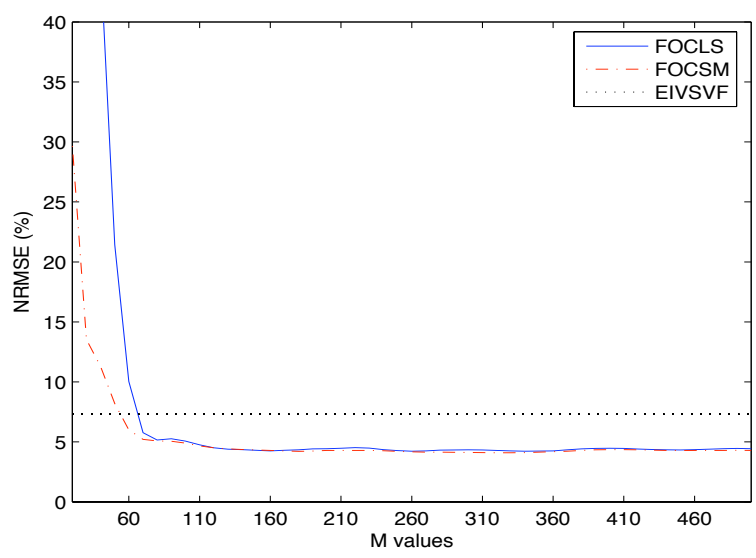

(b) White Gaussian noises, $N=5000$

Fig. 4. NRMSE as a function of the user parameter $M$.

\section{CONCLUSION}

In this paper, two new methods to consistently identify a continuous-time model in an EIV framework have been presented. The proposed methods are based on the use of fourth-order cumulants. The system is assumed to be excited by a non-Gaussian input and contaminated by Gaussian noises. As no other assumption is made on the measurement noises, they can be colored (and even mutually correlated), a case that cannot be handled by the few existing continuoustime EIV methods. Numerical simulations have illustrated the robustness of the proposed method to white and colored Gaussian noises.

\section{REFERENCES}

[1] J.C. Agüero and G.C. Goodwin. Identifiability of errors-in-variables dynamic systems. In 14th IFAC Symposium on System Identification, pages 196-201, Newcastle, Australia, March 2006.
[2] B.D.O. Anderson and M. Deistler. Identifiability in dynamic errors-invariables models. Journal of Time Series Analysis, 5(1):1-13, 1984.

[3] J.M.M. Anderson and W. Edmondson. System identification with noisy input/output data using cumulant-based Steiglitz-McBride algorithm. IEEE Transactions on Circuits and Systems II: Analog and Digital Signal Processing, 44(5):1021-1024, February 1997.

[4] J.M.M. Anderson and G.B. Giannakis. Noisy input/output system identification using cumulants and the Steiglitz-McBride algorithm. IEEE Transactions on Signal Processing, 44(4):1021-1024, April 1996.

[5] D.R. Brillinger. Time Series, Data Analysis and Theory. Holden Day, 1981.

[6] A. Delopoulos and G.B. Giannakis. Consistent identification of stochastic linear systems with noisy input-output data. Automatica, 30(8):1271-1294, 1994.

[7] B. Friedlander and B. Porat. Asymptotically optimal estimation of MA and ARMA parameters of non-Gaussian processes from highorder moments. IEEE Transactions on Automatic Control, 35(1):2735, January 1990.

[8] H. Garnier and L. Wang (Editors). Identification of continuous-time models from sampled data. Springer, 2007. To be published.

[9] H. Garnier, M. Mensler, and A. Richard. Continuous-time model identification from sampled data. Implementation issues and performance evaluation. International Journal of Control, 76(13):1337-1357, 2003

[10] J.-L. Lacoume, P.-O. Amblard, and P. Comon. Statistiques d'ordre supérieur pour le traitement du signal. Masson, 1997.

[11] E.K. Larsson, M. Mossberg, and T. Söderström. An overview of important practical aspects of continuous-time ARMA system identification. Circuits, Systems, and Signal Processing, 25(1):17-46, 2006.

[12] L. Ljung. System Identification: Theory for the User. Prentice Hall, 2nd edition, 1999.

[13] K. Mahata and H. Garnier. Identification of continuous-time errorsin-variables models. Automatica, 49(9):1470-1490, September 2006.

[14] J.M. Mendel. Tutorial on high-order statistics (spectra) in signal processing and system theory: theoretical results and some applications. Proceedings of the IEEE, 79(3):278-305, March 1991.

[15] C.L. Nikias and A.P. Petropulu. Higher-order spectra analysis. Signal Processing Series. Prentice Hall, Englewood Cliffs, 1993.

[16] G.P. Rao and H. Unbehauen. Identification of continuous-time systems. IEE Proceedings - Control Theory and Applications, 153(2):185-220, 2006

[17] T. Söderström. Errors-in-variables methods in system identification. In 14th IFAC Symposium on System Identification, pages 1-19, Newcastle, Australia, March 2006. Plenary Session.

[18] T. Söderström, E. Larsson, K. Mahata, and M. Mossberg. Using continuous-time modeling for errors-in-variables identification. In 14th IFAC Symposium on System Identification, pages 428-433, Newcastle, Australia, March 2006.

[19] T. Söderström, U. Soverini, and K. Mahata. Perspectives on errors-invariables estimation for dynamic systems. Signal Processing, 82:11391154, 2002.

[20] K. Steiglitz and L.E. McBride. A technique for the identification of linear systems. IEEE Transactions on Automatic Control, 43(10):23862397, October 1965.

[21] P. Stoica and T. Söderström. The Steiglitz-McBride identification algorithm revisited - convergence analysis and accuracy aspects. IEEE Transactions on Automatic Control, 26(3):712-717, June 1981.

[22] A. Stuart and J.K. Ord. Kendall's advanced theory of statistics, volume 1. Edward Arnold, Sixth edition, 1994.

[23] S. Thil, H. Garnier, and M. Gilson. A cumulant statistics-based method for continuous-time errors-in-variables model identification. In Workshop on Total Least Squares and Errors-in-Variables Modeling, Leuven, Belgium, 2006.

[24] J.K. Tugnait. Stochastic system identification with noisy input using cumulant statistics. IEEE Transactions on Automatic Control, 37(4):476-485, April 1992.

[25] J.K. Tugnait and Y. Ye. Stochastic system identification with noisy input-output measurements using polyspectra. IEEE Transactions on Automatic Control, 40(4):670-683, April 1995.

[26] E. Walter and L. Pronzato. Identification of Parametric Models from Experimental Data. Springer, 1997.

[27] P.C. Young. In flight dynamic checkout - a discussion. IEEE Transactions on Aerospace, 2:1106-1111, 1964.

[28] P.C. Young. Parameter estimation for continuous-time models - a survey. Automatica, 17(1):23-39, 1981 